\title{
Adaptation to contradictory information from vision and touch'
}

IRVIN ROCK, ARIEN MACK, LAURENCE ADAMS AND A. LEWIS HILL

YESHIVA UNIVERSITY

\begin{abstract}
Is proprioceptive information the source of adaptation to optically distorted visual stimulation? In an experiment where the only information as to optical minification of size is given by touch, there is no change in the visual perception of size. Rather the judgment of size via touch undergoes a transformation.

\section{Introduetion}

When vision is altered by experimental means, such as by prisms or lenses, any physical contact the observer has with objects could be thought to inform him of their true nature. Touch would remain veridical. It has therefore seemed plausible to many that visual adaptation is based on tactual information. Although there are good reasons for believing this argument to be incorrect a direct test of the thesis is nevertheless desirable. What is required is a situation where the only information available as to the optical distortion is provided by touch. This can be achieved by requiring the observer to view a single object through a lens or prism with nothing else visible, at the same time permitting him to touch or grasp the object.

\section{Method}

In the experiment reported here minification of size by a factor of two was the optical distortion studied. The observer grasped a thin wooden square from underneath, through black cloth, at the same time viewing it from above through a minification lens system. The method was generally similar to that previously employed by Fock \& Victor (1964).

What would be the effect of continued exposure to this conflict situation? Initially the square appeared to be half size when it was experienced by vision alone and even when simultaneously grasped it was erroneously judged to be about half its true size. Would the visual size begin to change as a result of the continual flow of veridical information from touch? Or would the opposite occur, namely that the signification of size via touch would begin to accommodate itself to the altered visual size?

Some means of keeping the observer occupied with a task during the exposure to the contradictory information was sought. In the method to be described here, the observer was required to match the standard square to one of a series of comparison squares all of which were seen through the minification lens. The comparison squares were mounted along the edge of a circular wheel which the observer could turn with his other hand, thus bringing one by one squares of varying size into the central region of the field. As each was moved into place the obserer grasped it and the standard alter- nately from underneath and thus, via vision and touch, compared the two. It is important to be clear that since both standard and comparison squares were minified and since both were grasped tactually, the observers had no difficulty comparing them and. $\mathrm{ky}$ and large, remained comoletely unaware that anythrng was amiss. Yet each time he grasped either square he was being exposed to a new relationship between fision and $60 \mathrm{wh}_{3}$ one which might have consequences for adaptation. The differences in size along the comparison series vere well above threshold. Several different size standards were used over a period of approximately $1 / 2 \mathrm{hr}$.

As a first step in the measurement of possible adaptive change, it was thought advisabie to determine if the cross-modality matching of size underwent any transformation or remained the same. If touch had succeeded in educating vision in this situation, ther the observer would have learned that a square whos appeared to be 1 in long actually was 2 in $3 m g$. This would be revealed by the selection $\hat{s} 2$ in tactual square to match a 1 in. visual square following the exposure period (a vision-touch or $\mathrm{V}-\mathrm{T}$ comparison) or conversely by the selection of a $1 / 2$ in visual square to match a 1 in tactual square (a towch -vision or T-V comparison). Presumably, prior to the exposure period, a 1 in visual square would be matched to a 1 in tactual square and vice versa. Both of these measures were obtained, in each case by taking 4 pre-exposure judgments and a post entura judgments. In the case of the comprestare was show a $2.7 \mathrm{~cm}$

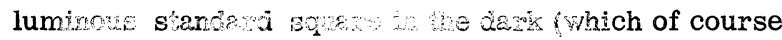
he did ato touch ane he hed to select a square experienced by touch alone $f_{2}$, not seen) to match the standard from a comparison series of squares on the wheel already referred to. In the case of the $\mathrm{T}-\mathrm{V}$ comparison, the subject grasped a $2.7 \mathrm{~cm}$ square (which of course he could not see) and he matched it to one of a series of visually presented luminous squares which were mounted along the edge of a circuiar wheel. The wheel was mounted on the wall at arm's length from the $S$. By turning the wheel the $S$ caused the comparison squares to appear one at a the in an opening cut in a black cloth covering the whee 1 . Eoth tactual and visual comparison series consisted of 11 squares which increased in size by steps of approximately $12 \%$. Square No. 6 , in the center of the series, was $2.7 \mathrm{~cm}$ thereby matching the stanciard. Ascending and descending trials were alternatied.

However, if only these two tests were employed the meaning of a change, if it occurred, would remain 
ambiguous. The same result would be predicted if vision had educated touch, namely to the effect that a square which felt to be 2 in on a side was actually only 1 in. Hence a method was needed which would indicate whether vision had undergone a change or whether touch had changed. It was decided to expose the observer to an object of a specific length and measure adaptation in terms of memory for that length. It has already been shown that this method is quite reliable (Rock, 1965). In the case of vision, the method consists in first presenting a $2.7 \mathrm{~cm}$ square with lights on for a brief period, then requiring the observer to select a luminous square in the dark to match the standard, from immediate memory. The visual comparison series described above was used. The sequence is repeated eight times, alternating ascending and descending presentations of the luminous comparison squares. This procedure serves to provide ample experience with the standard and a baseline against which to measure post-exposure judgments. Following the exposure period, in the dark, and without seeing the standard square again, the observer must select a luminous square which he believes is the size the standard square had been. The reason for obtaining the size measures in the dark is that otherwise the relative size of the square to all other visible objects would serve as a possible basis for judgment, thus working against an adaptive change. If visual size has undergone a tranformation in the predicted direction (and assuming memory for the phenomenal size of the standard is reasonably accurate) then the observer should select a smaller square after than before the exposure period. Four post-exposure judgments were obtained but because of a possible wearing off of an adaptation effect only the average of the first two of these judgments will be considered.

A similar method was employed to study the fate of touch perception. A $2.7 \mathrm{~cm}$ standard square was presented to the $S$ and he was allowed to see it while grasping it. Immediately thereafter he had to select a comparison square by touch alone which equalled the standard. The tactual comparison series described above was used. This sequence was repeated eight times. Following the exposure period he had to select a square by touch only, from memory, which he believed was equal in size to the previously felt standard square. If tactual size has undergone a change in the predicted direction, then the observer should select a larger square after the exposure period than before it. The reason why in this case the observer was initially allowed to see as well as grasp the standard was that in preliminary work it was discovered that memory for tactual size alone was quite imprecise and variable thus tending to obscure an adaptive change. By permitting vision of the standard the memory trace was apparently more definite and hence more stable. Since, however, the issue is not one of memory, but of perception via a given modality after exposure, the fact that in this condition the post-exposure test was conducted by touch alone guarantees that we are measuring." e size perception via that modality. The "before" and "after" tests both entail matching by touch alone.

\section{Results}

The results of all experiments are considered in terms of difference scores derived by comparing the mean of a S's first two post-exposure trial matches with the mean of his pre-exposure matches, the units being the steps along the comparison series. Two difference scores were computed, one based on the mean of the S's last two pre-exposure selections $\left(D_{1}\right)$ and one based on the mean of all pre-exposure selections $\left(D_{2}\right)$. For the V-T cross-modality procedure the average $D_{1}$ was +1.0 and the average $D_{2}$ was +1.05 , both of which are significantly different from 0 . An increase in the size of the tactual square selected is predicted. For the $\mathrm{T}-\mathrm{V}$ procedure the average $\mathrm{D}_{1}$ was -1.05 and the average $D_{2}$ was -.78, $D_{1}$ being significantly different from 0 and $D_{2}$ falling just short of significance at the .05 level. A decrease in the size of the visual square selected is predicted.

We turn now to the more direct measures of size perception in each modality. For vision, the average $\mathrm{D}_{1}$ was +.25 and $\mathrm{D}_{2}$ was +.16 , a nonsignificant change in the opposite direction from what would be predicted if size as signified by vision had been affected. For touch, the average $D_{1}$ was +.55 and the average $D_{2}$ was +.55 , these values differing significantly from 0 in the predicted direction. Since, however, the result was not as striking as in the $\mathrm{V}-\mathrm{T}$ or $\mathrm{T}-\mathrm{V}$ crossmodality comparisons (four Ss showing no change), the experiment was replicated with 10 new Ss. A virtually identical outcome was obtained, the mean difference scores $\left(D_{1}=+.60 ; D_{2}=+.64\right)$ being significantly different from 0 at approximately the .05 level. The combined data are convincing because for the $D_{1}$ scores $11 \mathrm{Ss}$ show a change in the predicted direction as compared to only 3 in the opposite direction (or 15 to 4 using the $\mathrm{D}_{2}$ scores)。

\section{Discussion}

The results of the four different tests support the conclusion that an adaptive change in the relationship of the tactual and visual modality to each other has taken place, and that that change is based on an altered signification of size via touch. Hence it would seem that in studies where an observer is exposed to optically created distortion of the visual image, it is not the case that adaptation is based on veridical information via touch.

\section{References}

Rock, I., \& Victor, J. Vision and touch: an experimentally created conflict between the two senses. Science, 1964, 143, 594. Rock, I. Adaptation to a minified image. Psychon. Sci., 1965, 2, 105-106.

\section{Note}

1. This research was supported by Grant GB-3410 from the National Science Foundation. 